\title{
Organizações de Saúde Intensivas em Conhecimento: um estudo no contexto de serviços de alta complexidade
}

\section{Health Organizations Based on Knowledge-intensive Work: a study in the context of high complexity services}

\author{
Cláudio Reis Gonçalo \\ Doutor em Engenharia de Produção. Professor do Programa de \\ Pós-Graduação em Administração da Universidade do Vale do \\ Rio dos Sinos. \\ Endereço: Av. Unisinos, 950 - UNISINOS - Centro de Ciências \\ Econômicas - Sala 5A406 São Leopoldo, RS, Brasil. \\ E-mail: cgoncaloœunisinos.br \\ Maria de Lourdes Borges \\ Mestre em Administração. Doutoranda em Administração pela \\ Universidade do Vale do Rio dos Sinos. \\ Endereço: Av. Unisinos, 950 - UNISINOS - Centro de Ciências Eco- \\ nômicas - Sala 5 A406 São Leopoldo, RS, Brasil. \\ E-mail: maluborg®gmail.com
}

\section{Resumo}

O objetivo deste estudo é identificar elementos críticos de gestão para promover a transformação de uma organização tradicional para uma organização de aprendizagem em serviços de alta complexidade em saúde. Assume-se que as organizações tradicionais são aquelas gerenciadas com ênfase na informação, e as organizações de aprendizagem são aquelas gerenciadas com ênfase no desenvolvimento de conhecimento. O conhecimento organizacional é reconhecido como um dos recursos de grande valor estratégico para a prestação de serviços de alta complexidade. A análise da gestão em serviços de saúde intensivos em conhecimento está estruturada a partir da qualificação do processo assistencial, o qual engloba as etapas de acesso, da permanência e da continuidade do tratamento. Realizou-se um estudo de caso em um hospital privado do Rio Grande do Sul, cuja estratégia de diferenciação é atuar na resolução de problemas de alta complexidade. Entre os elementos gerenciais críticos evidenciados salientam-se: que o paciente precisa ser compreendido como cliente; que o vínculo com a instituição não termina no final do atendimento hospitalar, mas somente na resolução do problema enfrentado com a doença; e que as especialidades precisam estar harmonizadas e dispostas a compartilhar conhecimento, impulsionadas por protocolos assistenciais reconhecidos por todos.

Palavras-chave: Conhecimento organizacional; Gestão em saúde; Administração hospitalar. 


\section{Abstract}

This study aims to identify critical management elements used to promote the transformation from a traditional organization to a learning organization, within the context of high complexity healthcare services. Traditional Organizations are based on the use of information, whereas Learning Organizations are based on knowledge creation. Organizational knowledge is viewed as a strategic resource for high complexity healthcare services. The analysis of the management of healthcare services based on knowledge-intensive work is structured through the qualification of the care process, which involves the stages of access, permanence and continuity of the treatment offered to patients. A case study was conducted in a private hospital of the state of Rio Grande do Sul, south of Brazil, whose strategy is to solve high complexity problems. Among the critical management elements that emerged, the main ones were: the patient should be viewed as a client; the Institution's contact with the patient should not terminate at the end of hospital assistance - only when the problem related to the disease is solved; and the medical specialties should be in harmony and willing to share knowledge, encouraged by assistance protocols that are recognized by all.

Keywords: Organizational Knowledge; Health Management; Hospital Administration.

\section{Introdução}

O objetivo deste estudo é identificar elementos críticos de gestão para a transformação de uma “organização tradicional” em uma “organização que aprende", no caso específico de serviços de alta complexidade em saúde. Considera-se como uma "organização tradicional” aquela que é gerenciada com ênfase na transferência de informação, e como uma "organização que aprende" aquela que é gerenciada para o favorecimento da transferência e criação do conhecimento (Alavi e Leidner, 2001; Von Krogh e Nonaka, 2001; Nonaka e col., 2006).

As organizações de saúde, reconhecidas como organizações intensivas em conhecimento, requerem que o conhecimento seja administrado como um recurso estratégico. Para tanto, as organizações necessitam implantar um processo contínuo de "garimpar" e "compartilhar" experiências, as quais são vivenciadas em todas as atividades participantes da cadeia de valor da prestação do serviço. Neste estudo, assume-se o pressuposto de que esse processo é uma alternativa estratégica emergente para que serviços de alta complexidade possam incrementar resultados com diferenciação.

A gestão sobre os recursos e a qualidade do atendimento no setor da saúde vem recebendo maior atenção devido ao aumento da complexidade do atendimento, bem como da ampliação dos sistemas de saúde (Boaden e Zolkiewski, 1998; Campbell e col., 2000; Bloom, 2008). Entretanto, as organizações de saúde necessitam estabelecer tanto estratégias mais eficazes, como modelos de gestão mais eficientes, para que incentivem o desenvolvimento do conhecimento como um recurso que agrega valor na cadeia de serviços e, em particular, que agrega valor para os pacientes (Porter e Teisberg, 2007).

Diante desse cenário, pressupõe-se que as organizações de saúde estão frente a um desafio emergente: a necessidade da definição de uma posição estratégica em relação à aquisição e transferência de conhecimento. A partir dessa opção estratégica assumida, as organizações poderão intensificar a obtenção de resultados oriundos da diferenciação de serviços ao longo da cadeia de valor. A organização hospitalar, dedicada à prestação de serviços complexos em saúde, se insere nessa necessidade de reflexão sobre sua mudança estratégica. Para contri- 
buir conceitualmente com esse problema gerencial de posicionamento estratégico, propõe-se para este estudo investigar a seguinte questão de pesquisa:

Como hospitais que prestam serviços complexos em saúde podem evoluir de uma "gestão tradicional", cujo foco é o acesso, o uso e a transferência de informação, para uma "gestão para aprendizagem", cujo foco é a transferência e criação de conhecimento?

Foi realizada uma pesquisa em um hospital do Rio Grande do Sul (Brasil) que definiu como opção estratégica ser reconhecido pela resolução de problemas de alta complexidade, possuindo 400 leitos e aproximadamente 2000 funcionários. A pesquisa apresenta uma abordagem qualitativa e utiliza a estratégia de investigação do estudo de caso. A unidade de análise para este estudo é o Centro Vascular, um dos serviços especializados da Instituição que foi a primeira da América Latina a oferecer, em uma única unidade, a prevenção, o diagnóstico e o tratamento das especialidades das doenças cardio e cerebrovasculares, de forma interdisciplinar e inovadora.

0 artigo apresenta, inicialmente, o referencial teórico sobre a gestão do conhecimento em serviços de saúde, incluindo a conceituação da gestão chamada de "Tradicional”, com ênfase em informação, e a gestão para "Organização que Aprende", com ênfase no conhecimento. A seguir, apresentam-se a metodologia de pesquisa, o estudo de caso, um modelo proposto para caracterizar elementos críticos de gestão para uma "Organização que Aprende" e, por último, as considerações finais.

\section{Gestão do Conhecimento em Serviços de Saúde}

Os conceitos analíticos de informação e de conhecimento estão imbricados, uma vez que informação é compreendida como forma de linguagem para comunicação explícita dos dados investidos de relevância e propósito, tornando-se um meio para construir conhecimento (Nonaka e Takeuchi, 1997; Davenport e Prusak, 1998; Sveiby, 2001). O conhecimento, por sua vez, é criado a partir do fluxo de informações comunicadas através da experiência ancorada em crenças e valores, tornando-se dinâmico e distinto, revelando-se nas experiências compartilhadas através das ações efetivas para o contexto apropriado (Nonaka e Takeuchi, 1997; Davenport e Prusak, 1998;
Sveiby, 2001; Williams, 2006). Observa-se, portanto, que a informação é percebida como objeto, como uma mídia para a comunicação explícita, enquanto o conhecimento manifesta o caráter processual, sendo recriado na capacidade para agir através da experiência pessoal (Sveiby, 2001).

Gestão do conhecimento em serviços de saúde significa alinhar pessoas, processos, dados e tecnologias para otimizar informações, colaboração, especializações e experiências com habilidade para impactar positivamente a performance organizacional. Assim, a gestão do conhecimento pode ocorrer em longo prazo e trazer um compromisso sustentável para a mudança da cultura nas organizações de saúde, tornando-as mais colaborativas, transparentes e pró-ativas (Guptill, 2005).

Os benefícios que a competição pode trazer ao setor da saúde são prejudicados quando o sistema valoriza a redução de custos. Assim, os prestadores e os planos de saúde (nos Estados Unidos) encontramse no emaranhado entre incentivos mal-alinhados e escolhas estratégicas infelizes (Porter e Teisberg, 2007). A única forma de transformar o sistema de saúde é realinhar a competição com o valor para o cliente. A cadeia de valor é uma proposição do ciclo de serviços, no qual naturalmente, para o fornecimento de qualquer produto ou serviço, ocorrem inúmeras atividades separadas. Para gerar valor à organização, essas atividades devem ser configuradas e integradas (Porter e Teisberg, 2007).

Os programas pay-for-performance $\left(\mathrm{P}_{4} \mathrm{P}\right)$ objetivam melhorar a qualidade de serviços de saúde através da utilização de incentivos financeiros para premiar médicos, hospitais e clínicas que entendam as especificações e metas de desempenho. 0 número de planos de saúde americanos que adotaram programas do tipo pay-for-performance $(\mathrm{P} 4 \mathrm{P})$ está crescendo rapidamente, mas a maioria encontra-se em processo de implementação (Rosenthal e Frank, 2005). Na Inglaterra, por exemplo, os pagamentos contribuem com 30\% da renda de algumas clínicas, mas na Califórnia, o montante recebido é bem menor (McDonald e Roland, 2009).

\section{A Transformação: da organização de saúde tradi- cional para a organização que aprende}

A literatura vem apresentando um grande interesse em estudos sobre a qualidade nas organizações de 
saúde e nos ambientes hospitalares (Aralsi e col., 2008; Collins e Joyce, 2008; Ruthes e Cunha, 2007; Borba e Kliemann Neto, 2008). Os programas de qualidade produziram uma mudança na filosofia gerencial das organizações de saúde, pois melhoraram a gestão dos recursos humanos, dos sistemas de informação, do desenho dos processos, do atendimento ao paciente e métodos de benchmarking (Johnson e Omachonu, 1995).

A revolução gerencial ocorrida a partir da gestão da qualidade foi, na essência, uma revolução a partir da mudança do acesso, do uso e da transferência da informação. A operação do modelo de gestão da qualidade é diretamente dependente dos sistemas de informação, os quais auxiliam na gestão da eficiência dos processos assistenciais. Considera-se neste estudo como "Tradicional” a organização de saúde que mantém o modelo de gestão com ênfase somente na informação. A gestão da qualidade tornou-se extremamente necessária, entretanto não suficiente.

Mesmo com os avanços proporcionados pela gestão da qualidade nas organizações de saúde e diante das mudanças globais que atingem todos os setores, o sistema de saúde precisa se transformar. Observa-se que é necessário mais empenho para explorar diferentes maneiras de produzir e entregar serviços, mais do que simplesmente intensificar esforços de recriar os existentes (Bloom, 2008), tal como é preconizado pela gestão da qualidade. O avanço significativo após a gestão da qualidade engloba a transformação para a "Organização de Saúde que Aprende".

Organizações de saúde que aprendem são pródigas em criar conhecimento. A essência do processo de criação do conhecimento ocorre em contextos e dispositivos favorecedores oferecidos pela organização (Nonaka e Takeuchi, 1997; Von Krogh e Nonaka, 2001). A aprendizagem organizacional ocorre de maneira mais incremental no aprimoramento das capacidades desenvolvidas e também na exploração de novas alternativas (March, 1991). As ações de melhoria que ampliam o conhecimento são implementadas nas organizações que aprendem para incrementar avanços estratégicos, levando em conta a natureza dinâmica do aprendizado organizacional em ambientes de incertezas (Fiol e Lyles, 1985; Crossan e col., 1999; March, 1991). Uma organização também aprende através de uma tensão saudável entre a certeza e a incerteza, em momentos como improvisação, pequenas vitórias e humor (Weick e Westley, 2004).

As organizações prestadoras de serviços de alta complexidade em saúde vêm se preocupando em compartilhar e adquirir conhecimento com os clientes e/ou consumidores. Organizações como Siemens Medical Solutions, Cerne e Solucient mantêm comunidades on-line com seus consumidores e utilizam as informações para continuamente renovar o conhecimento em seu produto, entrega e suporte (Guptill, 2005). 0 grupo farmacêutico japonês EISAI implementou o processo de criação do conhecimento como uma filosofia da gerência através do programa Human Health Care. Nesse programa, encontraramse no ambiente das pessoas doentes (famílias e cuidadores) outras formas de organizar o conhecimento e competências, por considerar que as competências científicas são parciais em relação às necessidades da sociedade (Fayard, 2003).

A base para a cocriação de valor nos serviços de saúde está na mudança de concepção na qual o paciente passa de recebedor passivo de tratamento para consumidor ativo de assistência médica (Prahalad e Ramaswamy, 2004). Para o sucesso dos cuidados com a saúde, Campbell e colaboradores (2000) pressupõem que os resultados do estado final do paciente, bem como do alívio de seus sintomas, sejam frutos - além dos precisos cuidados médicos, envolvendo relações sociais e psicológicas - de processos de interação entre o usuário e os profissionais, subsidiados em uma estrutura de recursos e gestão adequados.

\section{Metodologia}

Este estudo utilizou uma abordagem qualitativa, através de uma estratégia de investigação do estudo de caso (Yin, 2001). A unidade de análise foi uma unidade vascular, a qual pertence aos serviços especializados de um hospital privado de alta complexidade do Rio Grande do Sul (Brasil), com 400 leitos e aproximadamente 2000 funcionários. Este estudo é identificado como um caso revelador, pois apresenta a oportunidade de observar e analisar o fenômeno da estratégia organizacional para promo- 
ção de conhecimento em uma instituição que trata as doenças cardiovasculares e cerebrovasculares de forma interdisciplinar e inovadora. As expertises da Unidade Vascular centram-se no atendimento de Acidente Vascular Cerebral, Síndrome Coronariana Aguda, Doenças da Aorta e Doença Arterial Periférica.

A inserção no campo de pesquisa resultou em 43 visitas, realizadas de abril de 2006 a janeiro de 2008, e em 131 horas entre familiarização, observação e entrevistas. Isso foi necessário para identificar padrões recorrentes de eventos e/ou relacionamentos. Além das observações, foram realizadas 17 entrevistas, sendo seis delas com a alta administração. Foram entrevistados dois diretores da Instituição (hospital), um assessor da direção, a gerente da Universidade Corporativa, o diretor e o gerente administrativo da Unidade Vascular. Além dessas, foram realizadas sete entrevistas com o pessoal que trabalha na Unidade de Internação, englobando a gerência operacional e os profissionais que lidam diretamente com o cliente, tais como médicos, enfermeiras, técnicos de enfermagem e nutricionista. Deve-se salientar que o relacionamento entre o pesquisador e seus informantes precisa ser construído no desenvolvimento do estudo com muita sensibilidade e flexibilidade para a abertura ao diálogo e o sucesso da pesquisa (Leite e Vasconcellos, 2007).

No início da investigação, utilizou-se o Modelo de Gestão em Saúde proposto por Campbell e colaboradores (2000), representado pelos processos assistenciais de Acesso e Permanência na organização hospitalar. Após a coleta dos dados, procedeu-se a descrição das evidências utilizando-se a Análise de Conteúdo (Bardin, 1977). A partir das evidências encontradas e intenções estratégicas percebidas, construiu-se uma estrutura de análise de uma gestão voltada para aprendizagem, denominada Modelo Proposto, no qual foi incluído o processo assistencial de Continuidade.

\section{Apresentação e Análise do Caso}

A partir das evidências levantadas no estudo de caso exemplar, tornou-se possível identificar alguns procedimentos, ações e intenções que denotam as caracte- rísticas tanto da "Organização de Saúde Tradicional" como da "Organização de Saúde que Aprende".

\section{A organização de saúde tradicional}

No modelo da Organização de Saúde Tradicional (ênfase em informação), as diferenças de qualidade para o mesmo tratamento em organizações similares não são valorizadas nem pelo cliente nem pela fonte pagadora. Nesse modelo de remuneração, vigente na atualidade, ocorre uma disputa por quem vai pagar pelo quê entre os planos de saúde e as instituições. Ou seja, o resultado é buscado através da redução de custo. Isso gera um fracasso na competição, que não recompensa os melhores prestadores de serviço e os piores não vão à falência. Ocorre, assim, uma competição no nível errado e pelas coisas erradas (Porter e Teisberg, 2007). Conforme um dos entrevistados: "Isto representa uma miopia da fonte pagadora".

O plano de saúde não tem uma preocupação $e$ não consegue ter informação sobre o que está acontecendo com o paciente. Do ponto de vista de aderência ao tratamento [...] é uma miopia da fonte pagadora. (Entrevistado 1)

Se tu estás preocupado com a adesão ou não, isso é igual, do ponto de vista de concorrência de mercado, tu te preocupar com a adesão ao tratamento, ou tu não te preocupar, gera nenhuma diferença, significa que o serviço que tu presta depois, seja ele bom ou ruim, é igual. (Entrevistado 1)

Por isso, o valor financeiro para a organização, na estrutura tradicional, encontra-se na redução de custos, o que pode ser compreendido como um valor tangível. Esse aspecto está claro para o entrevistado EC4: "Hoje trabalha-se por redução de custo, pois isso não é ainda possível no Brasil, mas pode-se negociar no futuro um pay-for-performance, pagar por performance, por resultado". Tendência denominada $\mathrm{P}_{4} \mathrm{P}$ que objetiva melhorar a qualidade de serviços de saúde através de incentivos financeiros a prestadores de serviços de saúde que atendam as especificações e metas (McDonald e Roland, 2009).

\section{[...] Então se nós conseguirmos demonstrar para} uma fonte pagadora que os nossos pacientes que são egressos da Instituição têm bom..., boa satisfação, conseguem fazer programas de pre- 
venção adequadamente, então poderá haver um retorno financeiro para a Instituição. Então nós não estamos fazendo isso hoje, estamos hoje montando uma estrutura para que, no futuro, isso possa ser desenvolvido no Brasil, como está sendo desenvolvido em alguns lugares nos Estados Unidos. (Entrevistado 4)

O modelo Tradicional é baseado no serviço de internação, o qual está centrado em atendimentos focalizados em intervenções agudas e na permanência do paciente internado no hospital. 0 paciente é visto como passivo em uma relação de assimetria com o médico e com outros profissionais da saúde, conforme descrito por Prahalad e Ramaswamy (2004).

[...] Ninguém foi educado assim. Nós, como médicos, fomos educados para internar a Deus e todo mundo [...]. A cultura do atendimento altamente resolutivo acompanhado de ambulatório com resultados: isso nunca fez parte da noss a formação, nós fomos educados para ser um "doutorzão no consultório" [...] qual compromisso com o resultado? [...] Esse modelo que estamos propondo aqui é um modelo que gera muita antipatia. (Entrevistado 3)

Os médicos não são formados pra isso [acompanhamento], os médicos são formados pra fazer intervenção. O sistema pagador não está estruturado e organizado pra isso, ele está organizado pra pagar intervenção. Tudo isso tem seu modelo histórico e uma eficácia razoável. Para melhorar isso, precisamos mudar esse conceito. Esse conceito passa fundamentalmente por organizar, em primeiro lugar, os médicos, para funcionarem dessa maneira, para poder reeducar os pacientes, e forçar o modelo pagador a encontrar formas de remuneração desse serviço. (Entrevistado 1)

As especialidades clínicas são tratadas como separadas e independentes, e cada uma tem o seu 'domínio'. Há dificuldade de sistematização das informações, bem como para coletar e armazenar os dados e para gerar relatórios que possam embasar ações de melhoria. Atualmente, isso não é realizado de forma sistêmica. 0 conhecimento, basicamente, permanece no nível individual, e a comunicação com a equipe da Unidade de Internação ocorre de maneira informal entre o médico e os outros profissionais da saúde.
Para conseguir uma mudança de cultura é preciso se preparar para trabalhar 12 h por dia durante dez anos pra dizer: agora eu tenho resultados. [O principal impedimento cultural] é que ninguém foi educado assim. [...] A cultura do atendimento altamente resolutivo acompanhado de ambulatório com resultados: isso nunca fez parte da nossa formação. Eu tenho um tripé: acesso, permanência e continuidade. (Entrevistado 3)

O resultado da adesão do paciente é escassamente controlado, uma vez que existe pouca mensuração. Os estímulos para a adesão são realizados mediante recomendações multiprofissionais durante a internação. Nessas recomendações, são passadas informações para que o paciente as realize depois da alta hospitalar. Essas recomendações são realizadas do modo particular de cada profissional.

Para a organização de saúde baseada em informação, após a alta hospitalar, o paciente fica vinculado com o médico em seu consultório particular, o qual fica sendo a sua referência e não o hospital em que foi internado. Por isso, há uma fidelização do paciente ao médico. Os médicos são educados para trabalhar nesse tipo de modelo tradicional e são culturalmente voltados para a realização de intervenções.

\section{A organização de saúde que aprende}

No modelo da Organização de Saúde que Aprende (ênfase em conhecimento), a qualidade dos serviços prestados vai ao encontro da qualificação da assistência. Nessa qualificação a premissa da segurança para o cliente é garantida através de recursos, pessoas e processos em atendimentos de diferentes graus de complexidade e gravidade, conferindo eficácia no conjunto dos resultados. Isso está embasado no aprimoramento da capacidade de mensuração, é uma medicina de registros.

Estamos inaugurando essa linha de acompanhamento pós-alta [através de pesquisas]. Acompanhamento dos desfechos. [...] Se o hospital tem capacidade de seguir os pacientes pós-alta, ele vai ter informações, vai ter dados sobre o que está acontecendo, nós não sabemos isso, ninguém sabe no Brasil isso, pouca gente sabe. (Entrevistado 4) 
Nós temos hoje banco de dados, então o feedback de um banco de dados é muito importante, então o grande segredo da gestão médica é que nós temos que medir. É uma coisa muito simples, se conseguimos medir, podemos fazer gestão. Se nós não temos nenhuma mensuração nós não temos gestão. Então, por que estamos desenvolvendo essa plataforma de trabalho? Nós queremos ter seguimento dos nossos pacientes pós-alta. (Entrevistado 4)

O modelo que subsidia a "Organização que Aprende" é o Modelo Médico-Assistencial, o qual planeja, organiza e controla a gestão clínica do hospital estruturado em especialidades, sob a responsabilidade da Controladoria Médico-Assistencial. Pode-se compreender que o Modelo Médico-Assistencial representa o elemento crítico para a estratégia de conhecimento no sentido de agregar valor e vantagens competitivas (Wiig, 1997). As especialidades devem prestar contas assistenciais, de conhecimento e econômicas (resultado e de serviços), reguladas por contrato com os médicos.

A Instituição que não é capaz de gerar nada de conhecimento e, ao gerar, ela não é capaz de transferirnão é uma Instituição, é outra coisa. A criação do desenvolvimento desse conceito [criar a transferir conhecimento] dentro de casa está em pleno andamento. O problema é que todas as coisas que envolvem mudança de estrutura demoram pelo menos uma década. (Entrevistado 3)

A Instituição tem com um grupo de gestores um contrato, e esse contrato prevê prestação de contas assistenciais, prestação de contas econômicas e prestação de contas de conhecimento. Por exemplo, de quantas atividades científicas eles participaram, quantos artigos ou trabalhos eles apresentaram em congressos e prever para cada especialidade que protocolos sejam apresentados em uma Instituição do hospital que é a Controladoria Médico-Assistencial. (Entrevistado 1)

O modelo ambulatorial está centrado na proposta de assistência que possui um caráter de serviço preventivo e de atenção integral em regime de não internação, pois está estruturado para o atendimento de serviços menos complexos (Amaral, 1997; Brasil,
1987). A mudança de concepção estratégica inclui a adesão do cliente ao tratamento como uma consequência de toda a prestação de serviço, a qual ocorre na fase de continuidade do tratamento, subsidiada pelo serviço ambulatorial estruturado.

Hoje, todos os grandes processos de acreditação hospitalar estão baseados em três premissas: acesso, permanência e continuidade do tratamento. $\varepsilon$ na continuidade a questão da adesão é uma das coisas imensamente importantes. (Entrevistado 3)

Nós estamos falando do nosso processo-chave [adesão], nós estamos falando do core-business, esse é o maior valor possível que possa ser agregado, ou seja, o quanto eu posso contribuir diretamente para um paciente conseguir se recuperar, trazer aquele paciente para as condições de saúde ou de equilibrio. Mas ao mesmo tempo, fortalece a posição da Instituição de poder oferecer um produto à sociedade. Ou seja, se eu sei que consigo ter um produto que beneficie as pessoas, então o valor agregado éo meu produto latu sensu. Que é isso que as pessoas esperam. (Entrevistado 2)

Nesse modelo de serviços ambulatoriais, o paciente passa a ser visto como cliente a partir do vínculo que estabelece com o ambulatório, no qual realiza a continuidade do seu tratamento através de interações mais simétricas com o profissional de saúde. 0 paciente tem a possibilidade de discutir o diagnóstico e as recomendações, uma vez que o conhecimento médico encontra-se cada vez mais acessível pelos canais de comunicação e pela internet. 0 modelo ambulatorial é propício para o atendimento de pessoas com doenças crônicas, facilitando a cocriação de valor (Prahalad e Ramaswamy, 2004).

A fidelização do cliente tem muito a ver com o valor intangível. A fidelização é um valor para uma empresa. Se o paciente do instituto é bem atendido, isso cria um vínculo que gera satisfação para o cliente. [...] Isso é um diferencial do instituto. (Entrevistado 6)

$O$ cliente quer capacidade resolutiva e no momento que eu desenvolvo medicina, no momento que eu desenvolvo técnicas, no momento que eu coloco o conhecimento como o vetor para 
identificar mecanismos de solução pra ele, é isso que ele quer. Cliente não olha processo, cliente olha produto, então nós é que temos que, através desses mecanismos, garantir processos que ofereçam produtos com valor. O cliente quer isso, e o que garante esse produto é essa lógica. (Entrevistado 2)

O valor da organização passa a incluir o intangível, ou seja, o conhecimento passa a ser um dos maiores ativos da Instituição, valorizado como recurso estratégico e assim identificado na cadeia de valor. A adesão do cliente ao tratamento recomendado é o resultado do exercício da atividade segundo essa nova cadeia de valor (Porter e Teisberg, 2007). Essas ações são facilitadas se o cliente estiver vinculado ao ambulatório, em que comparece periodicamente para participação em grupos de acompanhamento pós-alta, realizando exames e consultas de rotina. Ou seja, o cliente está vinculado a um programa hospitalar para redução de eventos futuros.

Se o paciente estiver vinculado a um programa hospitalar que visa à redução de eventos, isso vai ser bom para o paciente, vai ser bom pra Instituição, porque os leitos hospitalares serão reservados para pacientes com eventos novos evai ser interessante também para a fonte pagadora, porque o custo desse paciente será menor. (Entrevistado 4)

Nesse modelo de "Organização que Aprende", o cliente reconhece a capacidade da instituição de resolver seus problemas, e como consequência ocorre o almejado bem-estar do cliente. A visão sobre o próprio bem-estar é o que o consumidor procura em um ambiente de assistência médica moderna em que participa ativamente de uma experiência de cocriação de valor (Prahalad e Ramaswamy, 2004). Nesse sentido, o cliente estabelece o vínculo com a instituição (através do ambulatório) e fica fidelizado à Instituição, e não ao médico e ao seu consultório.

Ambulatório é um sistema de atendimento que se destina a pessoas que deambulam, portanto, ambulatório é um sistema pra pacientes que têm condições de andar, não estão internados,

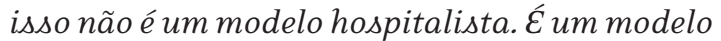
sistemista, é um sistema de atendimento. (Entrevistado 3)
O conhecimento é compreendido, portanto, em função do resultado para o cliente, pois é a via de geração das soluções, através de conceitos novos, da convergência e da transferência dos conhecimentos em uma equipe multiprofissional (Bukowitz e Williams, 2002). Esse posicionamento centrado em conhecimento deve trazer, além de resultados assistenciais, resultados financeiros através da fidelização do cliente.

O plano de saúde não tem uma preocupação e não consegue ter informação sobre o que está acontecendo com o paciente, do ponto de vista de aderência ao tratamento [...] é uma miopia da fonte pagadora. (Entrevistado 1)

Atualmente, a fonte pagadora não remunera ações que não estão dentro da permanência do cliente dentro do hospital, porém esta Instituição tem a intenção estratégica de reverter essa situação. Entretanto, essa estratégia é um investimento de médio e longo prazo. Reconhece-se que é um programa para redução de eventos no qual todos podem sair ganhando: a Instituição libera leitos para pacientes com alta complexidade; a fonte pagadora diminui o custo do paciente e economiza na sequência de eventos; e o paciente tem menos problemas devido à falta de adesão ao tratamento. Por isso, essa estrutura deveria ser remunerada por resultado.

Se o paciente estiver vinculado a um programa hospitalar que visa à redução de eventos, isso vai ser bom para o paciente, vai ser bom pra Instituição, porque os leitos hospitalares serão reservados para pacientes com eventos novos e vai ser interessante também para a fonte pagadora, porque o custo desse paciente será menor. Embora ele tenha que tomar mais remédio, diminui o custo por evitar novos eventos, novas internações, então estaremos fazendo uma medicina que tenha valor em si, uma medicina que, embora seja complexa e aparentemente custosa, ela tem seu valor porque economiza na sequência dos eventos. (Entrevistado 4)

No modelo da "Organização que Aprende", as fronteiras das especialidades tornam-se mais flexíveis, através de uma visão transdisciplinar, partindo da condição clínica e não da especialidade. Conforme explicação do entrevistado EC4: "Nós não fizemos cardiologia, neurologia... colocamos tudo isso dentro 
de um mesmo processo... este é o aspecto original que estamos desenvolvendo".

Acho que isso é a incorporação do conhecimento à prática médica, e no final há consequência para o paciente. Hoje em dia há menos angiografia, coloca-se menos stent revestido, e daqui a pouco vai ter um estudo que diz: olha, não é tão ruim assim e volta-se e chega-se a um ponto de equilíbrio. Então, do ponto de vista de conhecimento, e a cardiologia é muito pródiga para isso, eles estudam muito, estudam grandes grupos, tem muito dinheiro sustentando toda essa produção, ela não é exclusivamente acadêmica, muitos estudos são produzidos em função da necessidade de comercialização de droga. (Entrevistado 1)

Os processos de gestão do conhecimento enfocam as pessoas sobre práticas específicas que devem resultar em um produto particular, que servem para reabastecer, aumentar ou condensar a base de ativos do conhecimento conforme se observa no Instituto (Bukowitz e Williams, 2002).

\section{Análise geral a partir das evidências do estudo de caso}

Através da observação de que a maioria das organizações de saúde é Tradicional, para que ocorra a transformação em "Organização que Aprende", vários elementos constitutivos irão demandar mudanças de concepção. Em todo processo de mudança surgem dificuldades, especialmente quando tais mudanças relacionam-se à alteração do imaginário social, com relação ao processo coletivo de trabalho (Botti e Scochi, 2006). 0 quadro 1 resume as características das mudanças advindas deste estudo.

Ao assumir o consumidor como cliente, toda a estrutura organizacional deve estar orientada para conviver com um consumidor ativo, que espera por interações simétricas com profissionais cientes de seus direitos e bem informados sobre a sua doença. Para essa mudança de atitude frente ao cliente, a estrutura hospitalar adequada engloba em seu processo assistencial as dimensões de Acesso, Permanência e Continuidade do tratamento. Para apoiar essa transformação, é necessário inserir na atividade assistencial o valor ao conhecimento, reestruturando toda a cadeia de valor dos serviços prestados. A "Organização de Saúde que Aprende" se compromete com o cliente mesmo quando ele estiver fisicamente longe da organização, através do monitoramento das suas condições de saúde. A manutenção do contato remoto com o cliente pode fazer com que a solução do seu problema se torne uma das principais causas da eficácia organizacional.

\section{Modelo Proposto para a Organização que Aprende}

Propõe-se que a "Organização que Aprende" em serviços complexos de saúde seja estruturada no modelo de qualificação da assistência que engloba o Acesso, a Permanência e a Continuidade do tratamento oferecido aos clientes. Em um modelo similar (Campbell e col., 2000), consideraram as dimensões de Acesso e Efetividade, sendo que se propõe o desdobramento da dimensão Efetividade em Cuidado e Continuidade.

A segurança para o cliente é assegurada pela mobilização de recursos de alta complexidade, produzindo maior eficácia no conjunto dos resultados. Na figura 1 é apresentada a comparação entre as características da Gestão Tradicional (ênfase em informação) e da Gestão para Aprendizagem (ênfase em conhecimento) quanto às etapas da qualificação da assistência.

\section{0 acesso}

No modelo atual, aqui designado como "Tradicional" (ênfase em informação), o cliente tem acesso à Instituição hospitalar através da emergência do hospital ou por agendamento de procedimentos através dos consultórios médicos. No modelo da "Organização que Aprende" (ênfase em conhecimento), o acesso ao atendimento é realizado através da unidade ambulatorial da Instituição. Para o acesso, o ambulatório dispõe de acompanhamento remoto (a distância, por telefone ou via internet), bem como de consultas remotas. $\mathrm{O}$ ambulatório pode dispor de programas de pré-intervenção, para sensibilizar o cliente, aconselhamento e prevenção - através de grupos preventivos das doenças crônicas e contagiosas de maior morbimortalidade.

\section{A permanência}

A etapa da permanência na assistência é a etapa mais valorizada no modelo Tradicional, o qual pressupõe que a principal atribuição da organização de saúde é a realização de diagnóstico e da intervenção. Atualmente, nesse modelo, quando o paciente está internado, são passadas recomendações para que ele siga depois da alta hospitalar, sem nenhum tipo de 


\section{Quadro I - Elementos diferenciadores para uma Organização de Saúde que Aprende}

\begin{tabular}{|c|c|c|}
\hline Elemento & ORGANIZAÇÃO TRADICIONAL & ORGANIZAÇÃO QUE APRENDE \\
\hline Consumidor & PACIENTE & CLIENTE \\
\hline Modelo & $\begin{array}{l}\text { Hospitalista } \\
\text { Permanência do paciente, Intervenção/ } \\
\text { hospitalização }\end{array}$ & $\begin{array}{l}\text { Ambulatorial } \\
\text { Modelo Médico-Assistencial (Acesso, permanência e } \\
\text { continuidade) }\end{array}$ \\
\hline Valor & $\begin{array}{l}\text { Tangivel } \\
\text { Redução de Custos }\end{array}$ & $\begin{array}{l}\text { Intangível } \\
\text { Geração de novos conhecimentos através da gestão da } \\
\text { controladoria assistencial } \\
\text { Conhecimento aplicado na cadeia de valor }\end{array}$ \\
\hline Qualidade & $\begin{array}{l}\text { Diferenças de qualidade para o mesmo } \\
\text { tratamento não são valorizadas nem } \\
\text { pelo cliente nem pela fonte pagadora } \\
\text { (Porter e Teisberg, 2007) }\end{array}$ & $\begin{array}{l}\text { Qualificação da assistência, segurança para o cliente } \\
\text { Processos de Acreditação } \\
\text { Capacidade de mensuração, medicina de registros }\end{array}$ \\
\hline Especialidades & $\begin{array}{l}\text { Separadas e independentes } \\
\text { Dificuldade em armazenar informações, } \\
\text { coletar e armazenar dados }\end{array}$ & $\begin{array}{l}\text { Controladoria Médico-Assistencial } \\
\text { Controla e organiza a gestão clínica das especialidades } \\
\text { Contrato com modelo de gestão de prestação de contas, } \\
\text { renovação de protocolos }\end{array}$ \\
\hline Adesão ao tratamento & $\begin{array}{l}\text { Baixo controle/pouca mensuração, } \\
\text { Recomendações multiprofissional } \\
\text { durante a internação }\end{array}$ & $\begin{array}{l}\text { Resultado (feedback) do serviço } \\
\text { Alto controle } \\
\text { Consequência da cadeia de valor } \\
\text { Programa hospitalar para redução de eventos } \\
\text { Habilidades comunicacionais e interação dos profissionais com } \\
\text { o cliente são valorizadas para passagem do conhecimento }\end{array}$ \\
\hline Cliente & $\begin{array}{l}\text { Vínculo/fidelização com o médico } \\
\text { Referência no médico e no seu } \\
\text { consultório } \\
\text { Tratado como paciente }\end{array}$ & $\begin{array}{l}\text { Reconhece a capacidade da Instituição de resolver seus } \\
\text { problemas } \\
\text { Vínculo, além do médico, também com a Instituição/fidelizado } \\
\text { à marca }\end{array}$ \\
\hline Resultado & $\begin{array}{l}\text { Por redução de custos } \\
\text { Remuneração por intervenção } \\
\text { Fracasso na competição (fonte pagadora } \\
\text { não premia os melhores nem pune os } \\
\text { piores) } \\
\text { Miopia da fonte pagadora }\end{array}$ & $\begin{array}{l}\text { Retorno financeiro pela fidelização do cliente à Instituição } \\
\text { Consequência do Modelo Médico-Assistencial } \\
\text { Competição pela cadeia de valor baseada em conhecimento } \\
\text { Tentativa da instituição de reverter a situação através da } \\
\text { conquista de clientes institucionais }\end{array}$ \\
\hline Fonte pagadora & $\begin{array}{l}\text { Investimento de curto prazo } \\
\text { Remunera por intervenções emergenciais }\end{array}$ & $\begin{array}{l}\text { Investimento de médio e longo prazo } \\
\text { Proposição de programa hospitalar para redução de eventos } \\
\text { Pagamento por resultados atingidos }\end{array}$ \\
\hline Barreiras & $\begin{array}{l}\text { Modelo histórico } \\
\text { Médicos são culturalmente voltados para } \\
\text { intervenção em modelo hospitalista }\end{array}$ & $\begin{array}{l}\text { Modelo gera resistência dos profissionais da saúde } \\
\text { Resistência à mudança de estrutura }\end{array}$ \\
\hline
\end{tabular}


Figura I - Modelo de diferenciação entre estruturas de gestão em saúde.

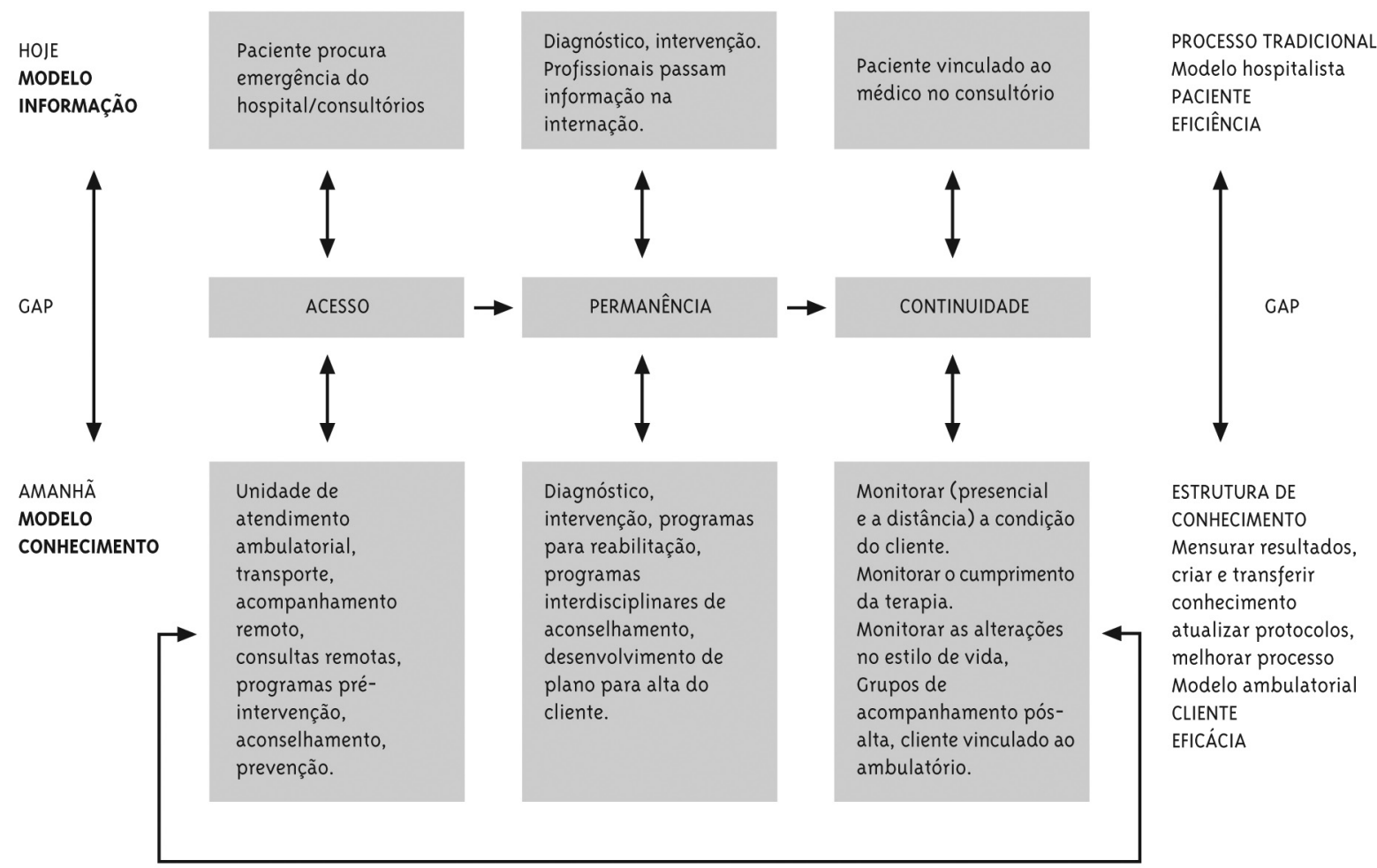

acompanhamento posterior, ficando o seguimento do tratamento sob sua única responsabilidade.

No modelo da Organização que Aprende, a etapa da permanência, além do diagnóstico, preparação e intervenção, envolve também programas para reabilitação, programas interdisciplinares de aconselhamento, desenvolvimento de plano para alta do cliente.

\section{A continuidade}

A etapa da continuidade na assistência é a etapa menos valorizada no modelo Tradicional, pois o seu foco está centrado na intervenção quando o paciente está internado no hospital. No modelo Tradicional, a continuidade do tratamento acontece no consultório particular, com o paciente vinculado ao médico. Assim, cabe ao médico passar as informações e controlar os resultados das recomendações realizadas. Na maioria das vezes, esse processo de controle não ocorre, e o paciente acaba voltando a procurar o médico ou a emergência do hospital, quando ocorrerem descompensações em função da não adesão ao tratamento recomendado.
No modelo da Organização que Aprende, a etapa da continuidade ao tratamento é muito valorizada, gerando a oportunidade de criar e transferir conhecimento a partir do acompanhamento da adesão ou não ao tratamento. A Organização que Aprende monitora a condição do cliente, seja de forma presencial ou a distância (através do telefone, celular ou mesmo através das ferramentas da internet), bem como o cumprimento da terapia e as alterações no seu estilo de vida. Além da monitoria a distância, o modelo da Organização que Aprende pode oferecer a atuação de grupos de acompanhamento pós-alta.

\section{Considerações Finais}

Este artigo se propôs a distinguir o conhecimento organizacional como valor estratégico para gestão de serviços em saúde e, em especial, serviços de alta complexidade. A partir de estudo de caso foi possível identificar elementos diferenciadores entre gestão Tradicional (com ênfase em informação) e 
gestão para Organização que Aprende (com ênfase em conhecimento).

A gestão para "Organização que Aprende" mostra-se como um desafio iminente para o setor da saúde, tanto no Brasil como no mundo, a qual sugere uma grande mudança de compreensão dos conceitos de competitividade e sobrevivência. Uma organização de saúde, cujo foco seja resolver problemas complexos altamente intensivos em conhecimento, necessita identificar estratégias de reconfiguração de suas atividades segundo uma nova cadeia interna de valor que também inclua o conhecimento como elemento diferencial.

O subsídio do conhecimento para a cadeia de valor atingir o sucesso gerencial indica, entre outras evidências, que o paciente precisa ser compreendido como cliente; que o vínculo com a instituição não termina no final do atendimento hospitalar, mas somente na resolução do problema enfrentado com a doença; e que as especialidades precisam estar harmonizadas e dispostas a compartilhar conhecimento, impulsionadas por protocolos médicos reconhecidos por todos.

Este estudo procurou contribuir para a reflexão sobre a mudança de posicionamento estratégico das organizações de saúde: da oferta de serviços que controlam a qualidade dos processos para a oferta de serviços que incluem também processos assistenciais a partir do conhecimento e de experiências vivenciadas. A cocriação de valor a partir das experiências dos relacionamentos com clientes, profissionais de saúde, parceiros, provedores e todos os envolvidos irá identificar oportunidades estratégicas de criação de conhecimento, propiciando a obtenção de melhores resultados dos serviços de alta complexidade em saúde.

\section{Referências}

ALAVI, M.; LEIDNER, D. E. Knowledge management and knowledge management systems: conceptual framework. MIS Quarterly, Minneapolis, v. 25, n. 1, p. 107-136, 2001.

AMARAL, M. Atenção à saúde mental na rede básica: estudo sobre a eficácia do modelo assistencial. Revista de Saúde Pública, São Paulo, v. 31, n. 3, p. 288-295, 1997.
ARASLI, H.; EKIZ, E.; KATIRCIOGLU, S. Gearing service quality into public and private hospitals in small islands. International Journal of Health Care Quality Assurance, Bradford, v. 21, n. 1, p. 8-23, 2008.

BARDIN, L. Análise de conteúdo. Lisboa: Edições 7O, 1977 .

BLOOM, G. Future health systems: why future? why now? Social Science \& Medicine, Nova Iorque, n. 66, p. 2067-2075, 2008.

BOADEN, R.; ZOLKIEWSKI, J. Process analysis in general practice: a new perspective? International Journal of Health Care Quality Assurance, Bradford, v. 11 n. 4, p. 117-122, 1998.

BORBA, G. S.; KLIEMANN NETO, F. J. Gestão hospitalar: identificação das práticas de aprendizagem existentes em hospitais. Saúde e Sociedade, São Paulo, v. 17, n. 1, p.44-6o, 2008.

BOTTI, M. L.; SCOCHI, M. J. O aprender organizacional: relato de experiência em uma Unidade Básica de Saúde. Saúde e Sociedade, São Paulo, v. 15, n. 1, p. 107-114, 2006.

BRASIL. Ministério da Saúde. Terminologia básica em saúde. 2. ed. Brasília, DF, 1987.

BUKOWITZ, W. R.; WILLIAMS, R. L. Manual de gestão do conhecimento. Porto Alegre: Bookmann, 2002.

CAMPBELL, S. M.; ROLAND, M. O.; BUETOW, S. A. Defining quality of care. Social Science \& Medicine, Nova Iorque, v. 51, p. 1611-1625, 2000.

COLLINS, C.; JOYCE, P. Focus on quality in healthcare in Ireland. International Journal of Health Care Quality Assurance, Bradford, v. 21, n. 2, p. 219-228, 2008.

CROSSAN, M.; LANE, H.; WHITE, R. An organizational learning framework: from intuition to institution. Academy of Management Review, Nova Iorque, v. 24, n. 3, p. 522-537, 1999.

DAVENPORT, T.; PRUSAK, L. Working knowledge: how organizations manage what they know. Boston: Harvard Business School, 1998. 
FAYARD, P. Comunidades estratégicas de conhecimento: uma proposta ocidental para o conceito japonês de Ba. Revista Famecos, Porto Alegre, n. 21, p. 20-29, ago. 2003.

FIOL, M.; LYLES, M. Organizational learning. Academy of Management Review, Nova Iorque, v. 10, n.4, p. 803-813, 1985 .

GUPTILL, J. Knowledge management in health care. Journal of Health Care Finance, Nova Iorque, v. 31, n. 3, p. 10-15, 2005.

JOHNSON, J.; OMACHONU, V. Total quality management as a health care corporate strategy. International Journal of Health Care Quality Assurance, Bradford, v. 8, n. 6, p. 23-28, 1995.

LEITE, S.; VASCONCELLOS, M. P. C. Construindo o campo da pesquisa: reflexões sobre a sociabilidade estabelecida entre pesquisador e seus informantes. Saúde e Sociedade, São Paulo, v. 16, n. 3, p. 169-177, 2007.

MARCH, J. Exploration and exploitation in organizational learning. Organization Science, Hanover, v. 2, n. 1, p. 71-87, 1991.

McDONALD, R.; ROLAND, M. Pay for performance in primary care in England and California: comparison of unintended consequences. Annals of Family Medicine, Leawood, v. 7, n. 2, p. 121-127, 2009.

NONAKA, I.; TAKEUCHI, H. Criação de conhecimento na empresa. Rio de Janeiro: Campus, 1997.

NONAKA, I., VON KROGH, G.; VOELPEL, S. Organizational knowledge creation theory: evolutionary paths and future advances. Organization Studies, London, v. 27, n. 8, p. 11791208, 2006.
PORTER, M., TEISBERG, E. Repensando a saúde: estratégias para melhorar a qualidade e reduzir os custos. Porto Alegre: Bookman, 2007.

PRAHALAD, C. K.; RAMASWAMY, V. O futuro da competição: como desenvolver diferenciais inovadores em parceria com os clientes. Rio de Janeiro: Campus, 2004.

ROSENTHAL, M. B.; FRANK, R. G. Early experience with pay-for-performance from concept to practice. JAMA, Chicago, v. 294, n. 14, p. 1788 1793, 2005 .

RUTHES, R.; CUNHA, I. Os desafios da administração hospitalar na atualidade. Revista de Administração em Saúde, São Paulo, v. 9, n. 35, p. 93-102, 2007.

SVEIBY, K. E. A knowledge-based theory of the firm to guide strategy formulation. Journal of Intellectual Capital, Bradford, v. 2, n. 4, p. 344-358, 2001.

VON KROGH, G.; NONAKA, I. Facilitando a criação do conhecimento: reinventando a empresa com o poder da inovação contínua. Rio de Janeiro: Campus, 2001.

WEICK, K.; WESTLEY, F. Aprendizagem organizacional: confirmando um oximoro. In: CLEGG, S.; HARDY, C.; NORD, W. Handbook de estudos organizacionais: ação e análise organizacionais. São Paulo: Atlas, 2004. v. 3, p. 361-388.

WIIG, K. M. Integrating intellectual capital and knowledge management. Long Range Planning, London, v. 30, n. 3, p. 399-405, 1997.

WILLIAMS, R. Narratives of knowledge and intelligence... beyond the tacit and explicit. Journal of Knowledge Management, London, v. 10, n. 4, p. 81-99, 2006.

YIN, R. K. Estudo de caso: planejamento e métodos. 2. ed. Porto Alegre: Bookman, 2001.

Recebido em: 10/10/2008

Reapresentado em: 03/09/2009

Aprovado em: 28/09/2009 